\title{
A comparison between S-N Logistic and Kohout-Věchet formulations applied to the fatigue data of old metallic bridges materials
}

\section{Joelton Fonseca Barbosa}

Federal University of Rio Grande do Norte, Natal, Brazil \& Federal Rural University of the Semi-Arid Region, Mossoró, Brazil Faculty of Engineering, University of Porto, Porto, Portugal

joeltonfb@gmail.com, https://orcid.org/0000-0003-3447-8240

José A.F.O. Correia, Pedro A. Montenegro

Faculty of Engineering, University of Porto, Porto, Portugal

jacorreia@inegi.up.pt,jacorreia@fe.up.pt, https://orcid.org/0000-0002-4148-9426

paires@fe.up.pt, http://orcid.org/0000-0001-5699-4428

Raimundo Carlos Silverio Freire Júnior

Federal University of Rio Grande do Norte, Natal, Brazil

freirej@ufrnet.br

\section{Grzegorz Lesiuk}

Faculty of Mechanical Engineering, Department of Mechanics, Material Science and Engineering, Wroctaw University of Science and Technology, Smoluchowskiego 25, 50-370 Wroctaw, Poland

Grzegorz:Lesiuk@pwr.edu.pl, bttps://orcid.org/0000-0003-3553-6107

Abílio M.P. De Jesus, Rui A.B. Calçada

Faculty of Engineering, University of Porto, Porto, Portugal ajesus@fe.up.pt, bttp://orcid.org/0000-0002-1059-715X

ruiabo@fe.up.pt, bttp://orcid.org/0000-0002-2375-7685

ABSTRACT. A new formulation of a Logistic deterministic S-N curve is applied to fatigue data of metallic materials from ancient Portuguese riveted steel bridges. This formulation is based on a modified logistic relation that uses three parameters to fit the low-cycle- (LCF), finite-life- and high-cyclefatigue (HCF) regions. This model is compared to the Kohout-Věchet fatigue model, which has a refined adjustment from very low-cycle fatigue (VLCF) to very high-cycle fatigue (VHCF). These models are also compared with other models, such as, power law and fatigue-life curve from the ASTM E739 standard. The modelling performance of the S-N curves was made using the fatigue data considering the stress fatigue damage parameter for the materials

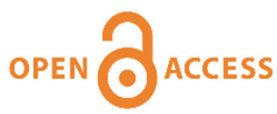

Citation: Barbosa, J., Correia, J., Montenegro, P., Júnior, R., Lesiuk, G., Jesus, A., Calçada, R., A comparison between S-N Logistic and Kohout-Věchet formulations applied to the fatigue data of old metallic bridges materials, Frattura ed Integrità Strutturale, 48 (2019) $400-410$.

Received: 22.10 .2018

Accepted: 27.12.2018

Published: 01.04 .2019 
from the Eiffel, Luiz I, Fão and Trezói riveted steel bridges. Using a qualitative methodology of graphical adjustment analysis and another quantitative using the mean square error, it was possible to evaluate the performance of the mean $\mathrm{S}-\mathrm{N}$ curve formulation. The results showed that the formulation of the S-N curve using the Logistic equation applied to the metallic materials from the article under the terms of the CC-BY 4.0, which permits unrestricted use, distribution, and reproduction in any medium, provided the original author and source are credited. old bridges resulted in a superior performance when compared with others models under consideration, both in the estimation of fatigue behaviour in the low-cycle fatigue (LCF) region and in the lowest mean square error.

KEYwORDS. Fatigue; Kohout-Věchet model; Fatigue-life curve; Prediction; Logistic formulation.

\section{INTRODUCTION}

7 he knowledge about the fatigue life expectancy of materials and structural components in engineering design is of great importance for the determination of load bearing during operation. Prediction of failure through mathematical and statistical modelling is a complex activity that attempts through an analytical model to consider the effects of cyclic stresses, stress intensity, predominance between traction and compression loading, frequency, number of experimental samples and the effects of manufacturing processes. Therefore, fatigue assessment is a difficult and still attractive challenge that remains an open problem in many situations.

One way to synthesize the number of factors involved in the fatigue life prediction is to reduce the model to the variables that are able to explain cause/effect, i.e. to fit a fatigue test to a stress level $\sigma_{i}$ (independent variable) to explain the number of cycles $N_{i}$ (dependent variable) required until failure. This fatigue model is known as S-N curves or Wöhler's curves, widely used in standards and standardization manuals such as ASTM E-739-10 [1], ISO 12107:2012 [2], EN 1993-1-9 [3], BS5400 [4], AASHTO [5], for engineering design of material and structural details. These standards are based on the Basquin equation [6,7] suggested in 1910, aiming at characterizing the fatigue behaviour in the high- (HCF) and low-cycle fatigue (LCF) regions.

Typically, fatigue data for preliminary design are studied in regions of $10^{3}$ to $10^{7}$ cycles. However, depending on the application, there is a need to prioritize estimation in the LCF or HCF regions. For extrapolation of estimates in HCF region, one must observe the adjustment equation and insert more fatigue data for regions of unknown space. For LCF region, the combination between the static strength and low-cycle fatigue data can be used to better fit the model. Concepts of engineering design for low-cycle fatigue regimes have been important to the use of advanced materials in different applications in mechanical designs. Civil engineering structures, railway and roadway bridges, offshore and ground structures, logistic structures, among others, are designed for the HCF regimes. Recently, a series of failures of these structures cannot be explained only with the HCF regime, taking into account the extreme loading conditions to which the structural elements are subject (e.g., earthquakes). Recent studies [8,9] suggest the use of S-N or $\varepsilon-\mathrm{N}$ curves covering both the LCF and HCF regimes.

The models used to estimate the S-N and $\varepsilon-\mathrm{N}$ curves, which have a good adjustment capacity in the low-cycle region, will provide greater reliability in estimating the fatigue damage parameters such as Smith-Watson-Topper, Strain, Walker-Like and energy-based criteria, among others. These approaches will allow the fracture mechanics to be able to predict crack onset by fatigue and residual life, more accurately. In addition, S-N curve with good adjustment in the low-cycle fatigue region allows the generalization of probabilistic fatigue models, and in this way, it is possible to estimate the load limit and reliability of the material or structural component at the beginning of the operation. The use of S-N curves in the fatigue life prediction can be related to fracture mechanics based approaches. Additionally, probabilistic approaches can be implemented to handle the uncertainties associated to the materials or models as observed in the research works [10-13] that have been applied and compared with existing fatigue data from the Portuguese riveted metallic bridges.

With the principle of meeting the most accurate estimates for the low-cycle fatigue regions, ranging from ultimate tensile strength to the high-cycle fatigue region, the Kohout-Věchet S-N curve is currently being proposed. This model has been increasingly used in fatigue life assessment of existing bridge structures $[6,14,15]$. Another model similar to Kohout-Věchet relation is the model proposed by $\mathrm{Mu}$ et al [16], in which a multi-slope model capable of adjusting the S-N curve in the three target regions, low-cycle-, finite-life- and high-cycle-fatigue, using a logistic function of three parameters is proposed. 
However, the analysis of Mu's work was limited to testing the model only for the T300/QY8911 carbon/epoxy composite. Knowing that this model has a wide adjustment capacity, in this research it will be applied to represent the fatigue behaviour of metallic materials from the ancient bridges, and in this way verify the performance of the S-N Logistic formulation.

A comparative study of the performance of the S-N adjustment equations, using models such as Kohout-Věchet, Logistic, ASTM and generalized Power law, will be applied to fatigue data from the old Portuguese riveted metallic bridges (Eiffel, Luiz I, Fão and Trezói). By means of a graphical adjustment analysis and the mean quadratic error, it will be possible to find the model that best fits with the experimental data. The results will be presented and discussed for a better recommendation on using the model in predicting fatigue of old bridges.

\section{METHODS USED IN THE MODELLING OF THE S-N CURVES}

Power Law

7 he generalized power law model is a derivation of the power law model of two terms, commonly used for the interpretation of fatigue data of composite and metallic materials. These models are of direct application and not based on any assumptions, even in limited databases. The estimation of the model parameters is based on the linear regression analysis that can be performed by simple calculations [17]. The generalized power law is given by Eqn. 1:

$$
\log \left(\sigma_{\max }\right)=A-B \log (N)^{C}
$$

where $\sigma_{\max }$ is the maximum stress amplitude parameter, $N$ is the number of cycles until the material failure, whereas $A, B$, and $C$ are the parameters of the fatigue model derived by linear regression analysis, resulting from the adjustment of the equation to the experimental data. The constant $C$ is an adjustment exponent that can smooth the S-N curve in the lowcycle fatigue region.

The S-N curve proposed by ASTM E739 standard ${ }^{1}$ [1] is widely used by researchers for their reliable and simple modelling process. This model does not recommend an extrapolation outside the experimental data region. The representation of the model can be done by linearized form (log-log) given by Eq. 2:

$$
\log (N)=A+B \log \left(\sigma_{\max }\right)
$$

The equations of the power law and ASTM E739 standard have similar structures for estimating the parameters of the curve in the linearized model $(\log -\log )$; however, these models have two relevant differences. The first difference presented by ASTM E739 standard when compared with the Power Law is to consider fatigue stress as a dependent variable, while the power law considers the fatigue stress an independent variable (the number of cycles to failure, $N_{f}$, is assumed as dependent variable). The second difference is the presence of a constant $C$, included in power law, able to smooth the fit in the lowcycle fatigue region.

\section{Logistic Function}

The logistic $S$-N curve model, developed by Mu [16], uses a logistic function to describe fatigue life behavior of composite materials, since this function is very similar to the $S$ shape, commonly observed in S-N curves (lin-log). The logistic function is adapted to model the S-N curve and is given by Eqn. 3:

$$
\sigma_{N}=\frac{1-c}{(1-a)+a e^{-b(\log N)}}+c
$$

where $a, b$ and $c$ are the material constants, obtained by nonlinear least squares, $\sigma_{N}$ is the normalized stress amplitude $\sigma_{N}=\sigma_{\max } / \sigma_{u l t}$ and $N$ is the number of cycles until failure. $\sigma_{u l}$ is the ultimate tensile strength.

\section{Kohout-Vèchet Model}

The full-amplitude S-N curve, based on the stress-damping parameter proposed by Kohout and Věchet, has been increasingly used in assessing the fatigue life of existing bridge structures [18]. The Kohout and Věchet S-N curve is a model based on geometric technical adjustment of fatigue behavior, based on stress or other damage linked parameter, that can 
achieve a good fit to the experimental data. The Kohout-Věchet fatigue model (KV) can estimate the behavior of the material in low-cycle (LCF) and high-cycle (HCF) regions [6], being able to cover the estimate from the ultimate tensile strength to the permanent fatigue limit. This KV model can be expressed by the following Eqn. 4:

$$
\sigma_{\max }=a\left[\frac{(N+B) C}{N+C}\right]^{b}
$$

where $a$ and $b$ are the similar to Basquin parameters, $B$ is the number of cycles corresponding to the intersection of the tangent line of the finite life region and the horizontal asymptote of the total tensile strength, and $C$ is the number of cycles corresponding to the intersection of the tangent line of the region of the finite life and the horizontal asymptote of the fatigue limit. The details for obtaining the parameters can be obtained in ref. [6].

\section{Normalized stress ranges}

The normalized stress ranges were suggested by Taras and Greiner [19] with aims to take into account the mean stress effects. The research work was developed for fatigue experimental results of riveted joints. This approach can be applied for fatigue results from metallic bridge materials to allow the comparison of experimental fatigue data from distinct mean stresses. The normalized stress ranges can be determined by

$$
\Delta \sigma_{\text {norm }}=\frac{\Delta \sigma}{f(R)}
$$

where, $\Delta \sigma_{\text {norm }}$ is the normalized stress range, $\Delta \sigma$ is the tested stress range, and $f(R)$ is a normalization function to account for stress ratio effects, defined as a function of the material.

For wrought/puddle iron and mild steel manufactured before 1900, $f(R)$ is defined as:

$$
\begin{aligned}
& f(R)=\frac{1-R}{1-0.7 \cdot R} \Leftarrow-1 \leq R \leq 0 \\
& f\left(R_{\sigma}\right)=\frac{1-R}{1-0.75 \cdot R} \Leftarrow R>0
\end{aligned}
$$

For mild steel after 1900, the normalization function to be used is the following:

$$
\begin{aligned}
& f(R)=\frac{1-R}{1-0.4 \cdot R} \Leftarrow-1 \leq R \leq 0 \\
& f\left(R_{\sigma}\right)=\frac{1-R}{1-0.6 \cdot R} \Leftarrow R>0
\end{aligned}
$$

However, the proposed normalization functions are only valid for high-cycle fatigue regimes, hence, they are not valid for low- and medium-cycle fatigue regimes. In this sense, fatigue design curves based on Goodman [20], Soderberg [21] and Gerber [22] diagrams become highly important for the fatigue life evaluation of old metallic bridges using local approaches.

\section{Comparison of THE Modeling Ability of the S-N Curve Formulations}

\footnotetext{
A comparison of the modelling performance of the S-N curves was made using the fatigue data of the materials from the ancient Portuguese riveted steel bridges (Eiffel, Luiz I, Fão and Trezói bridges). The performance of the mean fatigue curves was based on two methodologies of analysis. The first analysis the adjustment of the $\mathrm{S}-\mathrm{N}$ curve is based on direct graphical observation, where the degree of adjustment in the LCF and HCF regions is empirically assessed. The second analysis, quantitative, estimates the quality of the curve adjustment to the experimental fatigue data by means of the mean square errors (MSE). This parameter was calculated for each fatigue model by defining the error as the difference between the logarithms of the experimental and estimated values for the cyclic stresses, based on the following equation:
} 


$$
\text { MSE }=\frac{1}{n} \sum_{i=1}^{n}\left(\log \left(\sigma_{\text {exp }}\right)-\log \left(\sigma_{\text {est }}\right)\right)^{2}
$$

where $\sigma_{\text {exp }}$ and $\sigma_{e s t}$ are the experimental cyclic stress and the estimated cyclic stress levels of the S-N curves, respectively, for all models considered in this research. The stress levels correspond to the same number of cycles, $N$, of the experimental data. Therefore the comparison between fatigue models was evaluated qualitatively and quantitatively. Acquisition of each model was examined for its accuracy in modeling, ability to extrapolate and interpolate, number of model parameters, sensitivity to available experimental data, etc.

The MSE normalized calculation related with the adjustment of the presented S-N models to the experimental fatigue data, can be observed in Tab. 1. To facilitate the comparison between the S-N curve models, the results of the MSE values presented in Tab. 1 are normalized using the following expression

$$
\mathrm{MSE}_{\mathrm{NORM}}=\mathrm{MSE} / \mathrm{MSE}_{\mathrm{MAX}}
$$

where $\mathrm{MSE}_{\max }$ is the largest MSE (Equation 8) between the Logistic, Kohout-Věchet, Power Law and ASTM E739 models when compared by the bridge model.

\begin{tabular}{lccccc}
\hline \multirow{1}{*}{ Material } & \multicolumn{5}{c}{ Normalized mean squared error (MSE) } \\
& Logistic & Kohout-Věchet & Power Law & ASTM & $n$ \\
Eiffel $(\mathrm{R}=0)^{*}$ & 0.4946 & $\mathbf{0 . 4 9 4 3}$ & 0.5138 & 1 & 16 \\
Eiffel $(\mathrm{R}=-1)^{* *}$ & 0.6246 & 0.6741 & $\mathbf{0 . 6 2 1 4}$ & 1 & 27 \\
Luiz I $(\mathrm{R}=-1)^{* *}$ & $\mathbf{0 . 4 3 1 2}$ & 0.6450 & 0.4343 & 1 & 16 \\
Fão $(\mathrm{R}=0)^{* *}$ & 0.8618 & 0.9776 & $\mathbf{0 . 8 3 4 1}$ & 1 & 21 \\
Fão $(\mathrm{R}=-1)^{* *}$ & 0.5268 & $\mathbf{0 . 4 4 0 0}$ & 0.5705 & 1 & 14 \\
Trezói $(\mathrm{R}=-1)^{* *}$ & 0.5823 & 1.0000 & $\mathbf{0 . 3 9 6 0}$ & 0.8455 & 10 \\
All bridges $(\mathrm{R}=-1)^{* * *}$ & $\mathbf{0 . 6 6 8 2}$ & 0.7312 & 0.6788 & 1 & 66 \\
$*$ * Fatigue tests under stress-controlled conditions; ** Fatigue tests under strain-controlled conditions; *** Only bridges $\mathrm{R}=-1$. \\
\hline
\end{tabular}

Table 1: Normalized calculation of the mean squared error of the derived S-N curves.

The obtained S-N curve based on ASTM E739 standard did not present a satisfactory performance when compared with the other models, such as, Logistic formulation, Kohout-Věchet model and Power law. The fatigue model proposed by generalized Power Law obtained a smaller error in four of the seven fatigue data of the analyzed bridge materials, resulting in a better approximation of the S-N curve to the experimental data set. This method obtained the best estimate considering the MSE value computed for the material from the Trezoi bridge (see Table 1). The logistic model that uses only three parameters in the equation obtained a lower MSE value when all the experimental fatigue data are analyzed together, considering only the data of the fatigue tests under strain-controlled conditions at $\mathrm{R}=-1$. In the individual analysis of each bridge material it can be observed that the performance of the model was very similar to that of generalized Power Law model. For a set of fatigue experimental data with few data, it is possible to observe that some equations can't obtain such precise estimates. This situation is verified for the material from the Trezói Bridge at $\mathrm{R}=-1$ (fatigue test under straincontrolled conditions) with a sample of 10 specimens, where the Kohout-Věchet model obtained a very high MSE value when compared to Logistic formulation and Power Law. In general, the S-N curve using the Logistic formulation obtained satisfactory performance in terms of MSE values for all samples with size lower than 16.

In the low-cycle fatigue region (LCF), Logistic, Khout-Věchet and Power Law curves achieved better adjustments than the ASTM standard. This can be seen in Fig. 1 related with the material from the Eiffel bridge at $\mathrm{R}=0$ (fatigue test under stresscontrolled conditions), where these models are able to flatten and smoothen the inflection of the S-N curve in this region. 
For the materials from the Eiffel (Figs. 1 and 2) and Luiz I bridges (Fig. 3), it is possible to verify the robustness of adjustment of these formulations in the LCF region; in some cases the S-N curve becomes very close to the experimental values.

The improvement of the S-N curve in the low cycle region for Logistic, Power Law and Kohout-Věchet models is justified by the exponent of these equations, responsible for smoothing the rate of degradation in this region and the transition of elasto-plastic behavior.

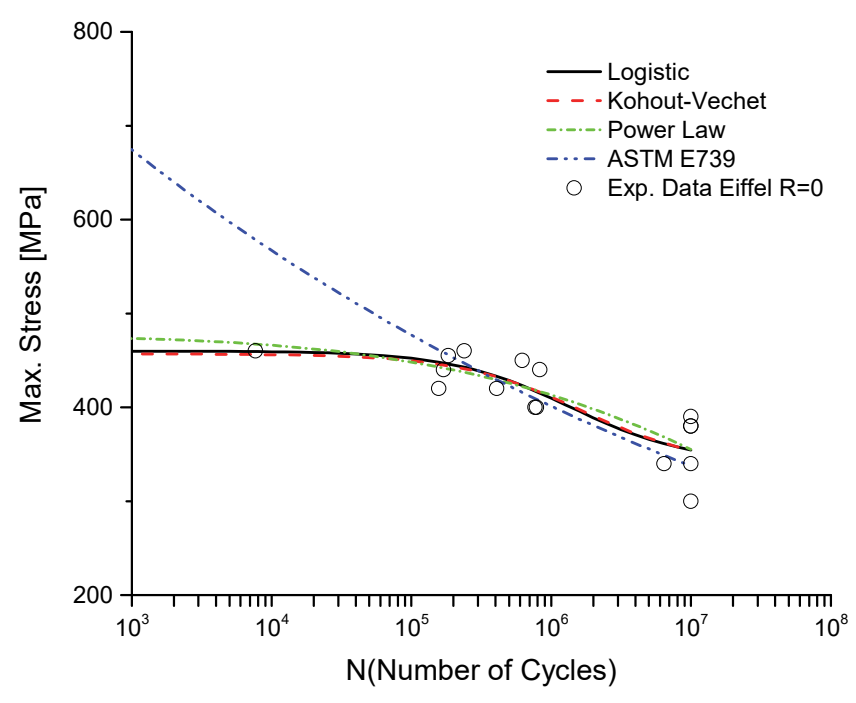

Figure 1: Comparison of the $\mathrm{S}-\mathrm{N}$ curves for the fatigue data of the metallic material from the Eiffel bridge, $\mathrm{R}=0$ (fatigue tests under stress-controlled conditions).

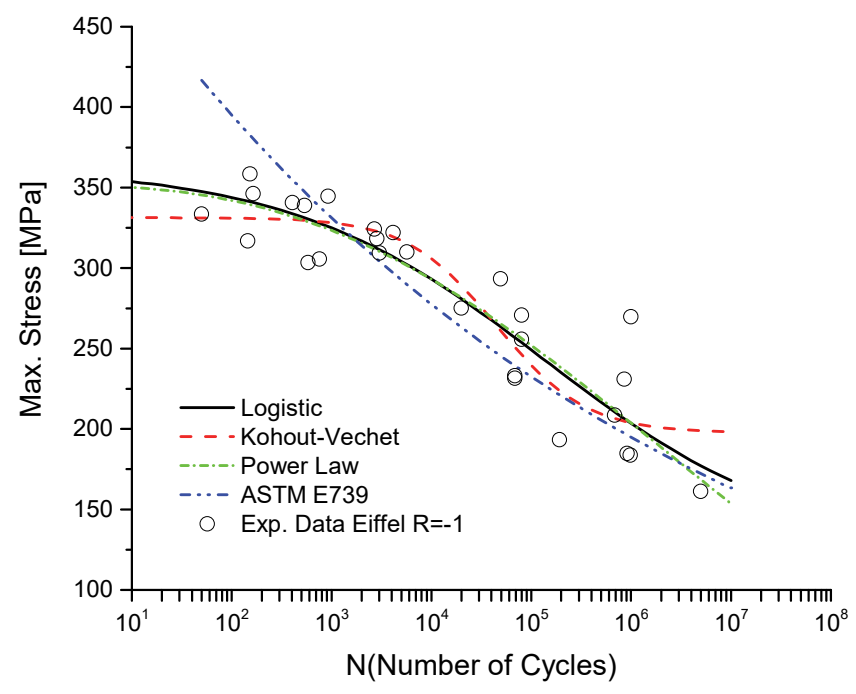

Figure 2: Comparison of the S-N curves for the fatigue data of the metallic material from the Eiffel bridge, $\mathrm{R}=-1$ (fatigue tests under strain-controlled conditions).

Fig. 2 presents the fatigue data of the material from the Eiffel bridge tested at $\mathrm{R}=-1$ (fatigue test under strain-controlled conditions), where it can be seen that the S-N curve based on ASTM E739 standard does not achieve a good approximation in the low-cycle fatigue region (LCF), even with data availability for the region smaller than $10^{3}$ cycles. In this region, the Logistic and Power Law curves performed slightly better than the Kohout-Věchet model. In the high-cycle fatigue region 
(HCF) the generalized Power Law curve presented a very pronounced degradation rate. The Kohout-Věchet model presented a coherent behaviour in the HCF region and are recommended to better indicate the fatigue limit of the material. Regarding the metallic material from Luiz bridge tested at $\mathrm{R}=-1$ (fatigue test under strain-controlled conditions), which can be seen in Fig. 3, the fatigue curves for the Power Law and Logistic model obtained very similar performance. The differences between them becoming more evident only in number of cycles higher than $10^{6}$. In the low-cycle fatigue region, for the Power Law, Logistic and Kohout-Věchet curves a very similar adjustment is observed. A poor performance of the S-N curve obtained from the Kohout-Vechet model for numbers of cycles to failure between $10^{5}$ and $10^{6}$ is verified, improving estimates to values above $10^{6}$. By analyzing the adjustement of the curve of the ASTM standard to the experimental data in the LCF region, it is verified an increasing distances between the experimental data and predictions, and consequently increasing the MSE value, making this model with the largest mean square error.

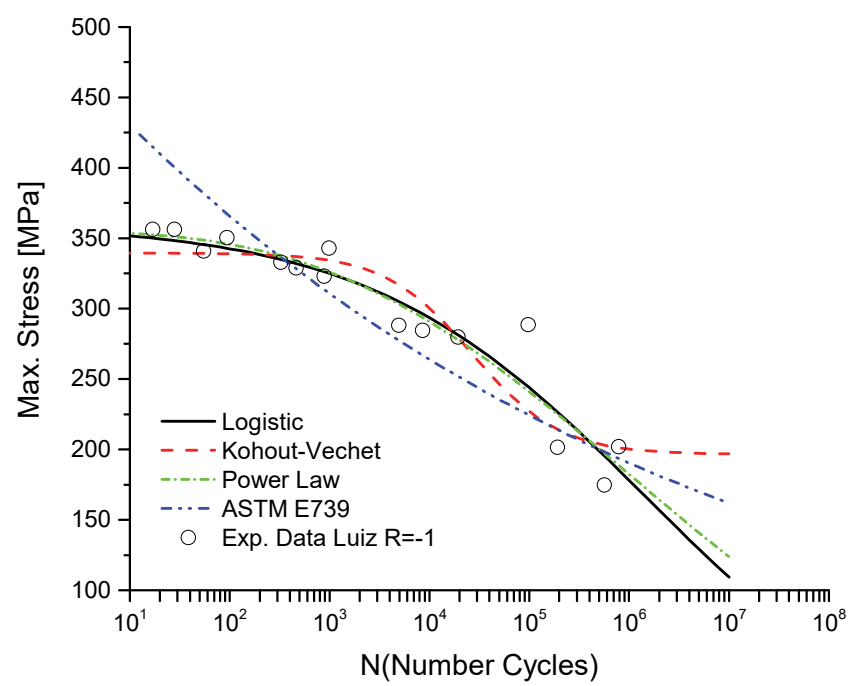

Figure 3: Comparison of the S-N curves for the fatigue data of the metallic material from the Luiz I bridge, $\mathrm{R}=-1$ (fatigue test under strain-controlled conditions).

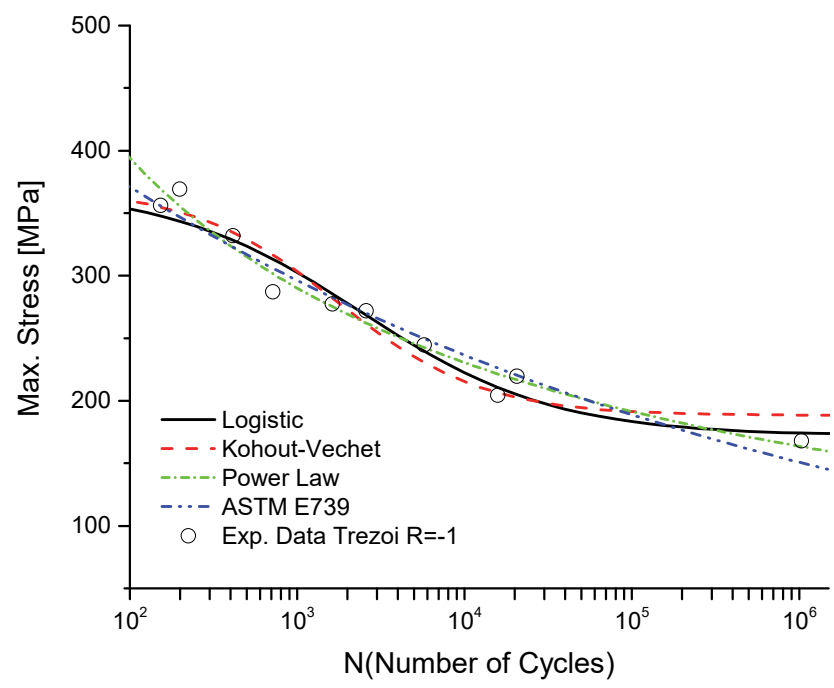

Figure 4: Comparison of the S-N curves for the fatigue data of the metallic material from the Trezói bridge, $\mathrm{R}=-1$ (fatigue test under strain-controlled conditions).

The available fatigue data from the material from the Trezoi Bridge consists on only 10 experimental points. Therefore, the conclusions to be obtained for the LCF and HCF regions are limited. Fig. 4 shows a marked difference in the fit of the analyzed S-N curves in the HCF region. The Power Law formulation obtained the lowest MSE (Tab. 1) followed by the Logistic model. These models achieved a good agreement with the experimental fatigue data above $10^{6}$ cycles. The ASTM standard and Kohout-Věchet methods did not provided good estimates in the HCF region. However, it is not possible to 
assert that the Logistic and Power Law formulations better describe the fatigue life behavior, due to the lack of data mainly in the high-cycle fatigue region. For a more complete analysis of this material, it is necessary to perform further experimental fatigue tests in stress levels between $400 \mathrm{MPa}$ and $300 \mathrm{MPa}$.

Regarding the fatigue data from the material of Fão bridge, two strain ratios, $\mathrm{R}$, were explored, namely $\mathrm{R}=0$ and $\mathrm{R}=-1$ (fatigue test under strain-controlled conditions). The S-N Kohout-Věchet formulation and Power Law resulted in the lowest MSE values and very close to each other. The only perceptible difference in a qualitative analysis of the S-N curves at fatigue strain ratio equal to 0 , is in the HCF region, where the possible extrapolation to the permanent fatigue limit of the Logistic S-N Curve would present a more adequate behaviour (Fig. 5).

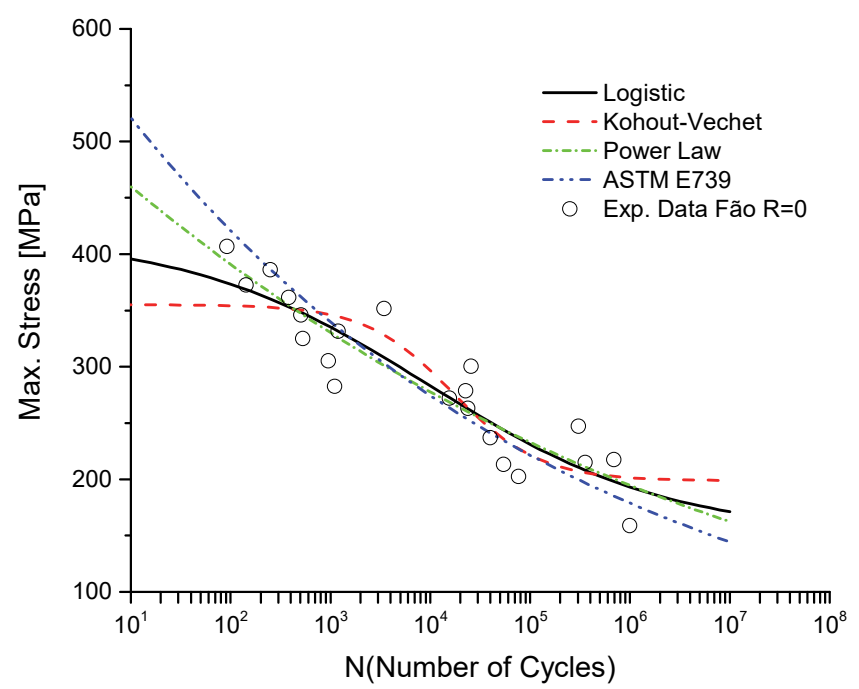

Figure 5: Comparison of the S-N curves for the fatigue data of the metallic material from the Fão bridge, $\mathrm{R}=0$ (fatigue test under strain-controlled conditions).

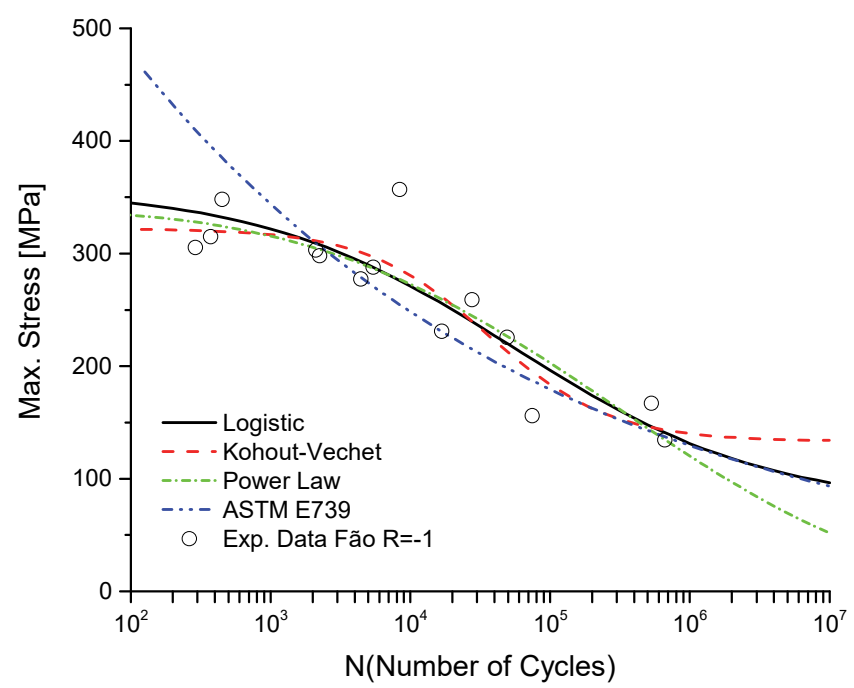

Figure 6: Comparison of the S-N curves for the fatigue data of the metallic material from the Fão bridge, $\mathrm{R}=-1$ (fatigue test under strain-controlled conditions).

In Fig. 6, regarding the fatigue data of the Fão bridge at $\mathrm{R}=-1$, the estimation in the LCF region of the Power Law, Logistic and Kohout-Věchet curves obtained similar and well-adjusted results, while in the HCF region the curve of Kohout-Věchet obtained better performance and consistent with fatigue limit.

Based on Figs. 5 and 6, it is possible to report that the Kohout-Věchet curve presents better performance in the HCF region justified by high MSE values. One the other hand, the adjustment results based on the ASTM standard leads to an adjustment not consistent with the experimental results and when compared with the other models. 


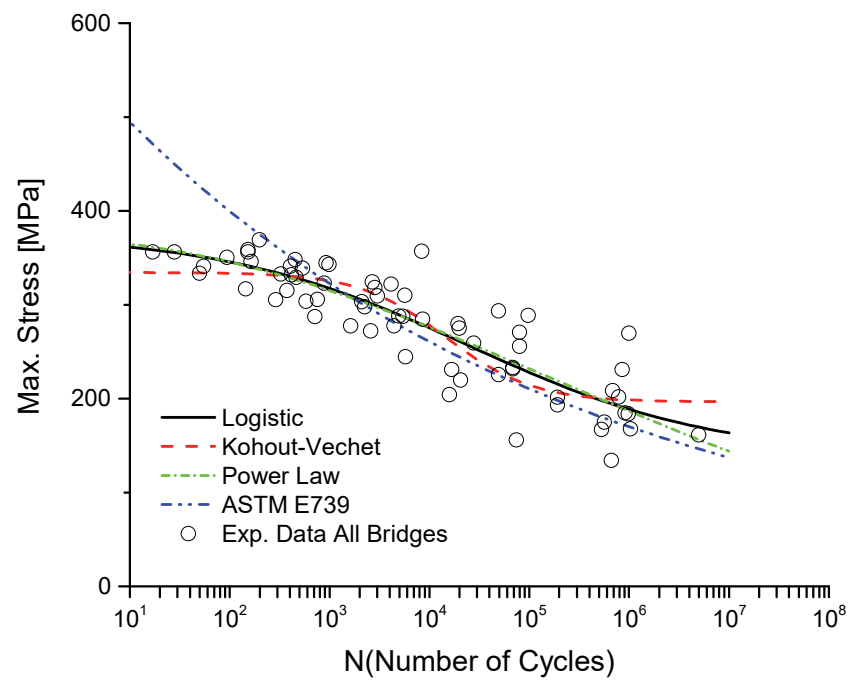

Figure 7: Comparison of the S-N curves for the fatigue data at $\mathrm{R}=-1$ (strain ratio) of the metallic materials from the all bridges.

Fig. 7 presents a comparison between the S-N curves generated based on different fatigue formulations, such as ASTM standard, Power Law, Logistic and Kohout-Věchet models, for the fatigue data at R=-1 (under strain-controlled conditions) of the metallic materials from the several bridges under consideration in this research.

A single S-N curve was estimated for each studied method considering a total of 66 experimental data available. These old metallic materials were extracted from members of the ancient metallic bridges of the 19th century, so this research sought to propose the method that is best suited to these materials. This analysis aims to consolidate what has already been observed in previous cases. The ASTM standard does not provide a good approximation in the low-cycle region even though experimental data are available in this region. The Power Law, Logistic and Kohout-Věchet S-N models obtained good adjustments to the low-cycle region when compated with the experimental data, as shown in Fig. 7. For the HCF region the Logistic, ASTM and Power Law methods presented a similar performance, however, only the Kohout-Věchet model didn't obtain a good agreement to the fatigue data in the region above $10^{6}$ cycles. In general the Logistic and Power Law method obtained the best fit according to the MSE estimation, however it is not conclusively due to the lack of data in regions above $10^{6}$ cycles.

\section{CONCLUSIONS}

he formulations of the S-N curves, using Logistic method, Kohout-Věchet model, Power Law and ASTM E739 standard, applied to the metallic materials of old bridges, obtained different performances mainly in the LCF and HCF regions. The ASTM standard does not perform well in estimating fatigue life in the low-cycle region. All analyzed graphs showed discrepant values of maximum stresses corresponding to the LCF region. Even following the recommendations of the ASTM E739 standard, of not extrapolating analysis beyond the experimental data, it is perceptible the difficulty of the method in approaching the LCF fatigue data. In the low-cycle region, the Logistic, Kohout-Věchet and Power Law methods presented satisfactory performance when compared with experimental data, however it is not possible to say which one has the best fit. A greater amount of experimental fatigue data would be needed in the LCF region to complete such analysis. In the high-cycle region, there is also a lack of experimental data, but assuming that the extrapolation of this region is expected to follow the permanent fatigue limit, it can be concluded that the Kohut-Vechet method presented better performance. The S-N curves of this model are distant from experimental fatigue data in regions above $10^{5}$ cycles. The generalized simple Power Law model can yield good approximations in the low-cycle region and in some cases in the region above $10^{5}$ cycles. The $\mathrm{S}-\mathrm{N}$ logistic curve formulation, which was initially applied only to composite materials, obtained an interesting performance when applied to the metallic materials of old bridges. In terms of MSE, this model obtained similar performance to the results of the Kohout-Věchet model, using only 3 parameters in the equation. Both models presented a good agreement with little experimental data. The largest difference between these models is in HCF region. 
The logistic model allowed a better approximation to the experimental data in HCF region and a graphical analysis showed better results for a possible extrapolation of the analysis.

The achieved results showed that the S-N curve formulation using the Logistic and Power Law equations obtained a better performance in the LCF and HCF regions and lower MSE values when compared to the generalized Power Law formulations and the ASTM E739 standard. It was also observed that the Logistic, Kohout-Verchet and Power Law equations are able to obtain smaller errors for the cases with a reduced number of experimental data. However, in order to generalize which model has a better fit, it is necessary to carry out an exhaustive study with a greater amount of experimental data of fatigue of other metallic materials.

\section{ACKNOWLEDGEMENTS}

his study was financed in part by the Coordenação de Aperfeiçoamento de Pessoal de Nível Superior - Brasil (CAPES) - Finance Code 001. The authors also acknowledge the Portuguese Science Foundation (FCT) for the financial support through the postdoctoral grant SFRH/BPD/107825/2015, as well as the funding of FiberBridge - Fatigue strengthening and assessment of railway metallic bridges using fiber-reinforced polymers (POCI-01-0145-FEDER030103) by FEDER funds through COMPETE2020 (POCI) and by national funds (PIDDAC) through Portuguese Science Foundation (FCT).

\section{REFERENCES}

[1] ASTM Committee and others. (2004). Standard Practices for Statistical Analysis of Linear or Linearized Stress-Life (S$\mathrm{N})$ and Strain-Life $(\varepsilon-\mathrm{N})$ Fatigue Data, ASTM Int. West Conshohocken, PA, USA.

[2] ISO, B.S. (2012). 12107: 2003, Metallic materials--Fatigue testing--Statistical planning and analysis of data, Int. Organ. Stand.

[3] Standard, E. (2003). Eurocode 3 : Design of steel structures, Part 1.9, Control, pp. 1-117.

[4] BSI (British Standards Institution). (1980). Steel, concrete and composite bridges. 10: Code of practice for fatigue, Eurocode 3, (1).

[5] Specifications, A.-L.B.D. (2004). American Association of State Highway and Transportation Officials (AASHTO), Washington, DC.

[6] Kohout, J., Vechet, S. (2001). A new function for fatigue curves characterization and its multiple merits, Int. J. Fatigue, 23(2), pp. 175-83.

[7] Weibull, W. (1961). Fatigue Testing and Analysis of Results: Publ. for and on Behalf of Advisory Group for Aeronautical Research and Development, North Atlantic Treaty Organisation, Pergamon Press.

[8] Chaminda, S.S., Ohga, M., Dissanayake, R., Taniwaki, K. (2007). Different approaches for remaining fatigue life estimation of critical members in railway bridges, Steel Struct., 7, pp. 263-76.

[9] Kajolli, R. (2013). A new approach for estimating fatigue life in offshore steel structures. University of Stavanger, Norway.

[10] De Jesus, A.M.P., Pinto, H., Fernández-Canteli, A., Castillo, E., Correia, J.A.F.O. (2010). Fatigue assessment of a riveted shear splice based on a probabilistic model, Int. J. Fatigue, 32(2), pp. 453-62, DOI: 10.1016/J.IJFATIGUE.2009.09.004.

[11]Jesus, A.M.P. de., Silva, A.L.L. da., Figueiredo, M. V., Correia, J.A.F.O., Ribeiro, A.S., Fernandes, A.A. (2011). Strainlife and crack propagation fatigue data from several Portuguese old metallic riveted bridges, Eng. Fail. Anal., 18(1), pp. 148-63, DOI: 10.1016/J.ENGFAILANAL.2010.08.016.

[12] Correia, J., Apetre, N., Arcari, A., De Jesus, A., Muñiz-Calvente, M., Calçada, R., Berto, F., Fernández-Canteli, A. (2017). Generalized probabilistic model allowing for various fatigue damage variables, Int. J. Fatigue, 100, pp. 187-194.

[13] Muniz-Calvente, M., de Jesus, A.M.., Correia, J.A.F.O., Fernández-Canteli, A. (2017). A methodology for probabilistic prediction of fatigue crack initiation taking into account the scale effect, Eng. Fract. Mech., 185, pp. 101-113, DOI: 10.1016/J.ENGFRACMECH.2017.04.014.

[14] Kohout, J., Vechet, S. (2008). Some Estimations of Tolerance Bands of SN Curves, Mater. Sci., 14(3), pp. $202-205$.

[15] Zapletal, J., Věchet, S., Kohout, J., Obrtlík, K. (2008). Fatigue lifetime of ADI from ultimate tensile strength to permanent fatigue limit, Strength Mater., 40(1), pp. 32-35.

[16] Mu, P.G., Wan, X.P., Zhao, M.Y. (2011). A New S-N Curve Model of Fiber Reinforced Plastic Composite, Key Eng. Mater., 462-463, pp. 484-8, DOI: 10.4028/www.scientific.net/KEM.462-463.484. 
[17] Freire Júnior, R.C.S., Belísio, A.S. (2014). Probabilistic S-N curves using exponential and power laws equations, Compos. Part B Eng., 56, pp. 582-90, DOI: 10.1016/j.compositesb.2013.08.036.

[18] Correia, J.A.F.O., Raposo, P., Muniz-Calvente, M., Blasón, S., Lesiuk, G., De Jesus, A.M.P., Moreira, P.M.G.P., Calçada, R.A.B., Canteli, A.F. (2017). A generalization of the fatigue Kohout-Věchet model for several fatigue damage parameters, Eng. Fract. Mech., 185, pp. 284-300, DOI: 10.1016/j.engfracmech.2017.06.009.

[19] Taras, A., Greine, R. (2010). Development and application of a fatigue class catalogue for riveted bridge components, Struct. Eng. Int. J. Int. Assoc. Bridg. Struct. Eng., DOI: 10.2749/101686610791555810.

[20] John Goodman. (1919). Mechanics Applied to Engineering, Longmans, Green, and co.

[21] Soderberg, C.R. (1930). Factor of Safety and Working Stress, Trans. Am. Soc. Test Matls, 52, pp. 146.

[22] Society of Automotive Engineers (1997). SAE Fatigue design handbook: AE-22. 